\title{
Global Suicide: A Problem Of Health Systems
}

OPINION

\begin{abstract}
Lucas Alves Araújo de Oliveira1,2, Lucas Fortes Portela Ferreira1,2, Racquel Brito da Silva 1,2, Sônia Isabel Romero de Souza ${ }^{3}$, Florido Sampaio das Neves Peixoto ${ }^{3}$, Jesus de Sousa Cartaxo ${ }^{3}$, Jorge André Cartaxo Peixoto ${ }^{3}$, Modesto Leite Rolim Neto ${ }^{1,2,3}$
\end{abstract}

\section{Abstract}

It is estimated that over 800000 people die by suicide and that there are many suicide attempts for each death. Young people are among those most affected. The numbers differ between countries, but it is the low- and middle-income countries that bear most of the global suicide burden, with an estimated $75 \%$ of all suicides occurring in these countries. The importance of each risk factor and the way it is classified will depend on each context. These factors can contribute to suicidal behaviours directly but can also contribute indirectly by influencing individual susceptibility to mental disorders. From the analysis of the rate of suicide, the numbers of suicide preventions successful with the number of hospitalizations and hospitalized for attempted suicide, it becomes possible to determine a path in order to create social change in three important factors to be followed to be fulfilled: knowledge (scientific and practical), public support (political will) and a social strategy, showing up as a national response to achieve the goals of suicide prevention.

\section{Keywords}

Global Suicide, Health Systems, Prevention
1 Medicine School, Federal University of Cariri -UFCA, Barbalha, Ceará, Brazil.

2 Research Group: Suicidology of Federal University of Ceará -UFC/ National Council of Scientific and Technological Development- CNPq.

3 Health Sciences Postgraduate Program, ABC Region Medical School, Santo André, São Paulo, Brazil.
It is a myth that people who talk about suicide do not mean to do it. People who talk about suicide may be reaching out for help or support [1]. A significant number of people contemplating suicide are experiencing anxiety, depression and hopelessness and may feel that there is no other option. In May 2013, the Sixty-sixth World Health Assembly adopted the first ever Mental Health Action Plan of the World health Organization (WHO) and the suicide is one of the most important topics, showing as an integral part of the plan, with the goal of reducing the rate of suicide in countries by $10 \%$ by 2020 [2]. 
This action plan and the accompanying resolution represent a formal recognition of the importance of mental health for WHO's 194 member states. It is also a commitment by all member states to take specified actions to improve mental health and to contribute to the attainment of a set of agreed global targets $[2,3]$.

It is estimated that over 800000 people die by suicide and that there are many suicide attempts for each death [1, 2]. Young people are among those most affected [1]. The numbers differ between countries, but it is the low- and middle-income countries that bear most of the global suicide burden, with an estimated $75 \%$ of all suicides occurring in these countries [2]. It is usual to think that only people with mental disorders are suicidal. Although, suicidal behaviour indicates deep unhappiness but not necessarily mental disorder [1]. Many people living with mental disorders are not affected by suicidal behaviour, and not all people who take their own lives have a mental disorder. Increasing evidence shows that the context is imperative to understanding the risk of suicide. Many suicides occur impulsively in moments of crisis and, in these circumstances, ready access to the means of suicide can determine whether a person lives or dies $[1,4]$.

The importance of each risk factor and the way it is classified will depend on each context. These factors can contribute to suicidal behaviours directly but can also contribute indirectly by influencing individual susceptibility to mental disorders [1, 5]. For example, "vulnerable groups" is used to refer to individuals or group of individuals who are made vulnerable by the situations and environments that they are exposed to [2]. These vulnerable group may be placed at a significantly higher risk of experiencing mental health problems, and includes minority groups, indigenous populations, older people, people experiencing discrimination and human rights violation, lesbian, gay, bisexual, and transgender persons, people exposed to conflict, natural disas- ter or other humanitarian emergencies $[2,5]$. There are other risk group to suicide behaviour, such as people who suffer from suicide bereavement. Some research's outcomes show that there is a increased risk of suicide in the 2 years after suicide of a partner compared with after death of partner from non-suicide causes [5].

In addition to the vulnerable groups, it is necessary to emphasize the presence of risk factors, which often are the ones who define the individual who attempt suicide, lives or dies [1]. Pesticides, firearms, hanging, easy access to high places, such as buildings and bridges, are means to realize a suicide attempt. Suicide by firearms account for the majority of suicides in some countries, such as the USA [6]. Available data show a close correlation between the proportions of households owning firearms and the proportion of firearm suicides [6, 7 , 8] Legislation restricting firearm ownership has been associated with a reduction in firearm suicide rates in many countries, including Australia, Canada, New Zealand, Norway and the United Kingdom. Besides, Pesticides account for an estimated one third of the world's suicides $[1,7,9]$. Suicide by intentional pesticide ingestion primarily occurs in rural areas of low- and middle-income countries (LMIC) in Africa, Central America, South-East Asia and the Western Pacific $[9,10,11]$.

There are multiple contributing factors and causal pathways to suicide and a range of options for its prevention. Usually no single cause or stressor is sufficient to explain a suicidal act [1]. Most commonly, several risk factors act cumulatively to increase an individual's vulnerability to suicidal behavior $[1,2]$. Some researches's outcomes show eight significant predictors of suicide remained in the final unconditional logistic regression model, in order of importance they were: high depression symptom score, previous suicide attempt, acute stress at time of death, low quality of life, high chronic stress, severe interpersonal conflict in the 2 days 
before death, a blood relative with previous suicidal behaviour, and a friend or associate with previous suicidal behavior [4]. These suicide risks increased substantially with exposure to multiple risk factors: none of the 265 deceased people who were exposed to one or fewer of the eight risk factors died by suicide, but 30\% (90/299) with two or three risk factors, 85\% (320/377) with four or five risk factors, and $96 \%(109 / 114)$ with six or more risk factors died by suicide [4]. Effective interventions are imperative as they can mitigate the risk factors identified $[1,4]$. Protective factors are equally important and have been identified as improving resilience. Therefore, enhancing protective factors is also an important aim of any comprehensive suicide prevention response [4].

It is necessary to know the distribuition's outlook of cases of suicide and attempted suicide to draw global protective factors that are effective in prevention. In 2012, suicide, occurred in $1.4 \%$ of all global deaths, setting the 15th leading cause of death [1, 2]. Worldwide, suicide is very common in the age group 15-29 years presenting itself as the second largest cause of death in this group [1, 2]. Men are the ones who most commit suicide [1, 2]. There is a variation in different countries regarding the prevalence of women1. The countries with lowaverage income are the most affected by suicide [1]. The age-standardized rate of suicide is somewhat higher in high-income countries than in low- and middle-income countries (LMICs) (12.7 versus 11.2 per 100000 population) [1]. However, given the much larger proportion of the global population that resides in LMICs, 75.5\% of all global suicides occur in these countries [1]. Among LMICs in the six WHO regions, there is an almost three-fold range in the age-standardized suicide rate, from a low of 6.1 per 100000 in the Region of the Americas to a high of 17.7 per 100000 in the South-East Asia Region [1]. One consequence of the different suicide rates in WHO regions is that in 2012 the South-East Asia
Region accounted for $26 \%$ of the global population but for $39 \%$ of global [1].

The suicide rate is a macro indicator of the population's psychosocial wellbeing and an evaluation criterion of the effectiveness of suicide prevention strategies [2]. Since suicide is a sensitive issue, and even illegal in some countries, it is very likely that it is under-reported. In countries with good vital registration data, suicide may often be misclassified as an accident or another cause of death [12]. A high level of injury deaths of undetermined intent (UD) is usually discussed in connection with the validity of suicide statistics [12]. There are some surveys that correlate this two rates to assess the validity of data on the number of suicides. The rates used can be found at WHO's data center [2]. This attempt to develop an approach to deal with the problem of "hidden suicides" (murders, accidents or injury death of undetermined intent erroneously classified as a suicide, or vice versa), proposing an appropriate reference point to assess the validity of the statistical suicide data, should be used to set targets in countries that hadn't reached the WHO's plan benchmark [2, 12].

All this global knowledge base about suicide and suicide attempts are needed to guide governments, policy makers and stakeholders. The Draft Comprehensive Mental Health Action Plan 2013-2020 comes with the ideation of practical measures for countries based on their contexts, and current resources, proposing a multisectoral approach in public health promotion and prevention of suicide. The WHO's priority is the presence of these actions on global agendas of public health and public policy, raising awareness suicide as a public health problem globally [2].

No one who is suicidal is determined to die. Suicidal people are often ambivalent about living or dying [2]. Someone may act impulsively by drinking pesticides, for instance, and die a few days later, even though they would have liked to live on [2]. 
Suicides are preventable. Access to emotional support at the right time can prevent suicide [2].

This support is shown in the plan, in three different stages: universal prevention strategies are designed to reach an entire population in an effort to maximize health and minimize suicide risk by removing barriers to care and increasing access to help, strengthening protective processes such as social support and altering the physical environment; selective prevention strategies target vulnerable groups within a population based on characteristics such as age, sex, occupational status or family history - while individuals may not currently express suicidal behaviours, they may be at an elevated level of biological, psychological or socioeconomic risk-; indicated prevention strategies target specific vulnerable individuals within the population - e.g. those displaying early signs of suicide potential or who have made a suicide attempt [2].

In countries where suicide prevention activities have not yet taken place, the emphasis is on seeking out stakeholders and developing activities where there is greatest need or where resources already exist1. It is also important to improve surveillance at this stage [1]. In countries with some existing suicide prevention activities, a situation analysis can show what is already in place and indicate where there are gaps that need to be filled1. Countries that already have a relatively comprehensive national response should focus on evaluation and improvement, updating their knowledge with new data and emphasizing effectiveness and efficiency [1].

National strategies for suicide prevention should follow the options of implementation plan of the WHO, which include: increase public, political and media awareness of the magnitude of the problem, and the availability of effective prevention strategies; restrict access to the means of self-harm and suicide (for instance, firearms and pesticides); access and manage self-harm or suicide and associated mental, neurological and substance use disorders (as outlined in the mhGAP intervention guide); optimize psychosocial support from available community resources both for those who have attempted suicide as well as for families of people who have committed suicide [2].

For these actions take effect and be improved, it is necessary to feed the databases because these data are still scarce and not very reliable for prevention. From the analysis of the rate of suicide, the numbers of suicide preventions successful with the number of hospitalizations and hospitalized for attempted suicide, it becomes possible to determine a path in order to create social change in three important factors to be followed to be fulfilled: knowledge (scientific and practical), public support (political will) and a social strategy, showing up as a national response to achieve the goals of suicide prevention [1]. 


\section{References}

1. WHO. Preventing suicide: a global imperative. Geneva: World Health Organization, 2014.

2. WHO. Mental health action plan 2013-2020. Geneva: World Health Organization, 2013.

3. WHO. mhGAP intervention guide for mental, neurological and substance use disorders in non-specialized health settings. Geneva: World Health Organization, 2010.

4. Phillips MR, Yang G, Zhang Y, Wang L, Ji H, Zhou M. Risk factors for suicide in China: a national case-control psychological autopsy study. Lancet 2002; 360: 1728-1736.

5. Pitman A, Osborn D, King M, Erlangsen A. Effects of suicide bereavement on mental health and suicide risk. Lancet Psychiatry 2014; 1: 86-94.

6. Brent DA, Bridge JA. Firearms availability and suicide: Evidence, interventions, and future directions. Am Behav Sci. 2003; 46(9): 1192-1210.

7. Anglemyer A, Horvath T, Rutherford G. The accessibility of firearms and risk for suicide and homicide victimization among household members: a systematic review and meta-analysis. Ann Intern Med. 2014; 160(2): 101-10.

8. Ajdacic-Gross V, Killias M, Hepp U, Gadola E, Bopp M, Lauber $C$ et al. Changing times: a longitudinal analysis of international firearm suicide data. Am J Public Health. 2006; 96: 1752-5.

9. Gunnell D, Eddleston M, Phillips MR, Konradsen F. The global distribution of fatal pesticide self-poisoning: systematic review. BMC Public Health. 2007; 7: 357.

10. Eddleston M, Karalliedde L, Buckley N, Fernando R, Hutchinson $\mathrm{G}$, Isbister $\mathrm{G}$ et al. Pesticide poisoning in the developing world a minimum pesticides list. The Lancet. 2002; 360(9340): 1163-7.

11. Gunnell D, Fernando R, Hewagama M, Priyangika WDD, Konradsen F, Eddleston $\mathrm{M}$. The impact of pesticide regulations on suicide in Sri Lanka. In J Epid. 2007; 36: 1235-42.

12. Värnik $P$, Sisask $M$, Värnik $A$, et al. Massive increase in injury deaths of undetermined intent in ex-USSR Baltic and Slavic countries: hidden suicides?. Scand J Public Health 2010; 38 (4): 395-403.

\section{Comment on this article:}

\section{(f) [in $8+\mathbf{S}$.}

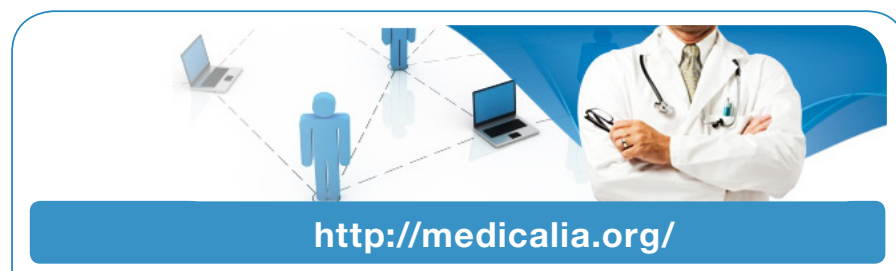

Where Doctors exchange clinical experiences, review their cases and share clinical knowledge. You can also access lots of medical publications for free. Join Now!

\section{Publish with iMedPub}

http://www.imed.pub

International Archives of Medicine is an open access journal publishing articles encompassing all aspects of medical science and clinical practice. IAM is considered a megajournal with independent sections on all areas of medicine. IAM is a really international journal with authors and board members from all around the world. The journal is widely indexed and classified Q1 in category Medicine. 